\title{
Synthesis and Structure of Ladder Oligosilsesquioxanes: Tricyclic Ladder Oligomethylsilsesquioxanes
}

\author{
Ken-ichi Suyama, Takahiro Gunji, Koji Arimitsu, Yoshimoto Abe *
}

Department of Pure and Applied Chemistry, Faculty of Science and Technology, Tokyo

University of Science, 2641 Yamazaki, Noda, Chiba, 278-8510, Japan, R\&D Center, Teraoka Seisakusho Corporation, 1-4-22 Hiromachi, Shinagawa-ku, Tokyo 140-8711, Japan

abeyoshi@rs.noda.tus.ac.jp 


\section{Molecular structure of $5 \mathrm{c}$}

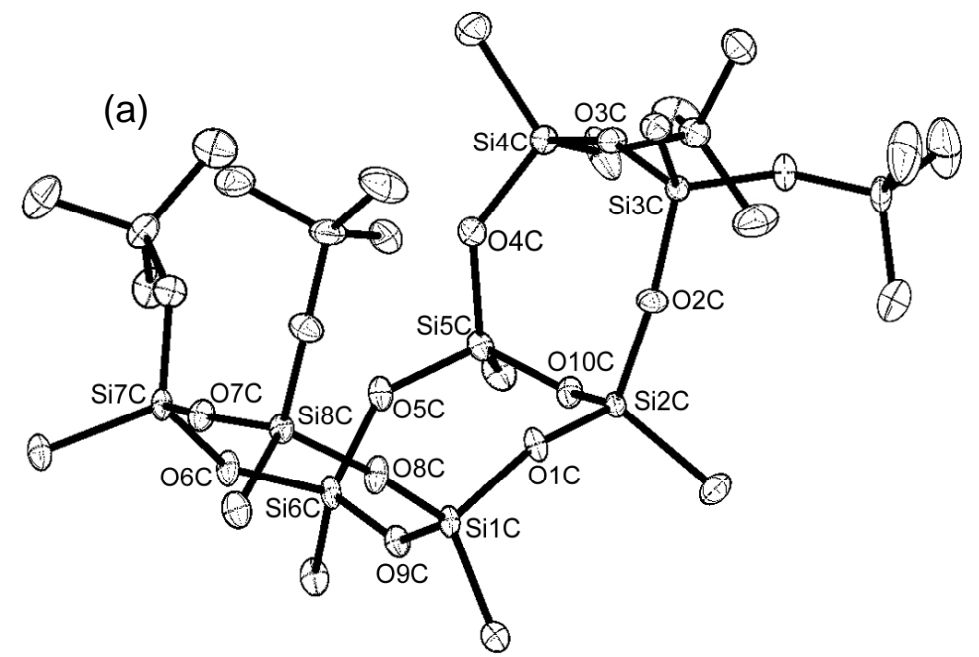

(b)

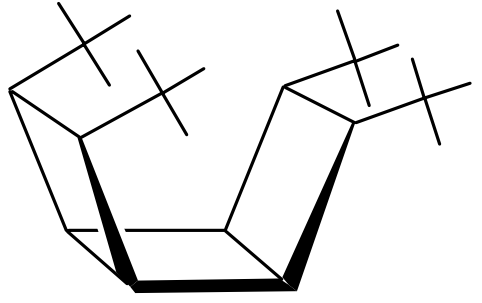

Figure S1. ORTEP drawing (a) and schematic drawing (b) of 5c. Thermal ellipsoids are drawing at the $30 \%$ probability level. Hydrogen atoms are omitted for clarity. The average bond lengths and bond angles were $1.62 \AA$ for $\mathrm{Si}-\mathrm{O}, 155.4^{\circ}$ for $\mathrm{Si}-\mathrm{O}-\mathrm{Si}$, and $109.0^{\circ}$ for O-Si-O.

Figure $\mathrm{S} 1$ shows that $\mathbf{5 c}$ is constructed of a ladder framework with three eight-membered rings where the central ring Si1C-Si2C-Si5C-Si6C combines with the side rings Si1C-Si6C-Si7C-Si8C and Si2C-Si3C-Si4C-Si5C at dihedral angles of $54.3^{\circ}$ and $54.0^{\circ}$, respectively. Thus the structure of $\mathbf{5 c}$ was also determined to be a syn-configuration ladder oligosilsesquioxane. The average bond lengths and angles of the central eight-membered ring were $1.62 \AA$ for $\mathrm{Si}-\mathrm{O}, 148.6^{\circ}$ for $\mathrm{Si}-\mathrm{O}-\mathrm{Si}$, and $109.6^{\circ}$ for O-Si-O. These parameters of siloxane bond are similar to those of $\mathbf{5 a}$. The two $t$-butoxy groups of $\mathbf{5} \mathbf{c}$ bonded to the silicon atoms $\mathrm{Si}(3 \mathrm{C}, 4 \mathrm{C})$ and $\mathrm{Si}(7 \mathrm{C}, 8 \mathrm{C})$ on each side ring are arranged in cis configuration, with two being located on the plane of the central eight-membered ring while the others are outside the central ring, so that steric hindrance due to the $t$-butoxy groups decreases compared with that of $\mathbf{5 a}$.

Although the molecular structure of $\mathbf{5 c}$ was determined as noted above, there are many alerts notes in the CIF-check files. As shown in Table S1, the X-ray crystallographic data definitely suffered from its poor refinement: triclinic $\mathrm{P}-1$ space group derivative has $\mathrm{Z}=2(\mathrm{~V}=8495.1)$, whereas isomeric 5a crystallizing in the same space group has $Z=2(V=2118.7)$. This definitely evidenced for the existence of the four independent molecules in the unit cell of 5c. These four molecules seem to be not totally identical in their geometries and moreover some carbon atoms are represented by the large thermal ellipsoids. For this reason, the refinement of the structure of $\mathbf{5 c}$ is far from the ideal. Perhaps, the poor quality of the crystal is the major problem of the refinement. 
Table S1. Crystallographic Data for $5 a, 5 c$, and $5 d$

\begin{tabular}{|c|c|c|c|}
\hline & 5a & $5 c$ & 5d \\
\hline formula & $\mathrm{C}_{24} \mathrm{H}_{60} \mathrm{O}_{14} \mathrm{Si}_{8}$ & $\mathrm{C}_{24} \mathrm{H}_{60} \mathrm{O}_{14} \mathrm{Si}_{8}$ & $\mathrm{C}_{24} \mathrm{H}_{60} \mathrm{O}_{14} \mathrm{Si}_{8}$ \\
\hline formula weight & 797.44 & 797.44 & 797.44 \\
\hline crystal system & Triclinic & Triclinic & Triclinic \\
\hline space group & P-1 & $\mathrm{P}-1$ & $\mathrm{P}-1$ \\
\hline $\mathrm{a}, \AA$ & $8.6034(16)$ & $13.7590(11)$ & $8.5654(14)$ \\
\hline $\mathrm{b}, \AA$ & $13.100(2)$ & $17.1192(13)$ & $13.721(2)$ \\
\hline $\mathrm{c}, \AA$ & $19.985(4)$ & $38.575(3)$ & $19.278(3)$ \\
\hline$\alpha, \operatorname{deg}$ & 103.091(3) & $102.5820(10)$ & $76.599(2)$ \\
\hline$\beta$, deg & $101.413(4)$ & $91.6040(10)$ & $78.407(2)$ \\
\hline$\gamma, \operatorname{deg}$ & $96.798(4)$ & $105.8170(10)$ & $83.239(2)$ \\
\hline $\mathrm{V}, \AA^{3}$ & $2118.7(7)$ & $8495.1(12)$ & $2153.0(6)$ \\
\hline Z & 2 & 8 & 2 \\
\hline$\mu, \mathrm{mm}^{-1}$ & 0.333 & 0.306 & 0.302 \\
\hline theta range, deg & 1.08 to 28.46 & 1.47 to 27.93 & 1.53 to 27.93 \\
\hline independent reflections & 10340 & 38166 & 10054 \\
\hline reflections collected & 15064 & 50687 & 27810 \\
\hline solution & SHELXL-97 & SHELXL-97 & SHELXL-97 \\
\hline$R 1$ & 0.0580 & 0.0812 & 0.0554 \\
\hline$W R 2$ & 0.1729 & 0.1899 & 0.1225 \\
\hline S & 1.104 & 1.025 & 1.153 \\
\hline No. of params & 435 & 1657 & 435 \\
\hline
\end{tabular}




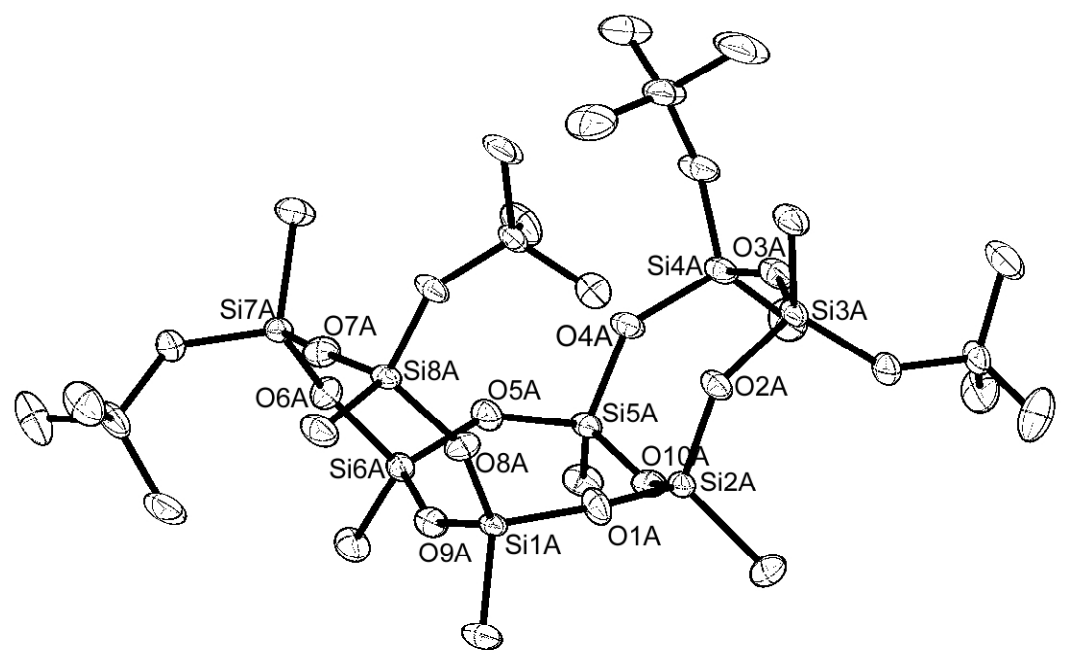

Figure S2. ORTEP drawing of 5a. Thermal ellipsoids are drawing at the $50 \%$ probability level. Hydrogen atoms are omitted for clarity.

Table S2. Selected bond lengths and angles of 5a

\begin{tabular}{llll}
\hline \multicolumn{5}{c}{ Bond lengths $(\AA)$} \\
$\mathrm{Si}(1 \mathrm{~A})-\mathrm{O}(1 \mathrm{~A})$ & $1.6125(17)$ & $\mathrm{Si}(1 \mathrm{~A})-\mathrm{O}(8 \mathrm{~A})$ & $1.6121(16)$ \\
$\mathrm{Si}(1 \mathrm{~A})-\mathrm{O}(9 \mathrm{~A})$ & $1.6163(19)$ & $\mathrm{Si}(2 \mathrm{~A})-\mathrm{O}(1 \mathrm{~A})$ & $1.6161(17)$ \\
$\mathrm{Si}(2 \mathrm{~A})-\mathrm{O}(2 \mathrm{~A})$ & $1.6245(17)$ & $\mathrm{Si}(2 \mathrm{~A})-\mathrm{O}(10 \mathrm{~A})$ & $1.6306(19)$ \\
$\mathrm{Si}(3 \mathrm{~A})-\mathrm{O}(2 \mathrm{~A})$ & $1.6284(17)$ & $\mathrm{Si}(3 \mathrm{~A})-\mathrm{O}(3 \mathrm{~A})$ & $1.631(2)$ \\
$\mathrm{Si}(4 \mathrm{~A})-\mathrm{O}(3 \mathrm{~A})$ & $1.623(2)$ & $\mathrm{Si}(4 \mathrm{~A})-\mathrm{O}(4 \mathrm{~A})$ & $1.6273(18)$ \\
$\mathrm{Si}(5 \mathrm{~A})-\mathrm{O}(4 \mathrm{~A})$ & $1.6183(17)$ & $\mathrm{Si}(5 \mathrm{~A})-\mathrm{O}(5 \mathrm{~A})$ & $1.6219(17)$ \\
$\mathrm{Si}(5 \mathrm{~A})-\mathrm{O}(10 \mathrm{~A})$ & $1.6262(19)$ & $\mathrm{Si}(6 \mathrm{~A})-\mathrm{O}(6 \mathrm{~A})$ & $1.6117(17)$ \\
$\mathrm{Si}(6 \mathrm{~A})-\mathrm{O}(5 \mathrm{~A})$ & $1.6226(17)$ & $\mathrm{Si}(6 \mathrm{~A})-\mathrm{O}(9 \mathrm{~A})$ & $1.6252(19)$ \\
$\mathrm{Si}(7 \mathrm{~A})-\mathrm{O}(6 \mathrm{~A})$ & $1.6199(16)$ & $\mathrm{Si}(7 \mathrm{~A})-\mathrm{O}(7 \mathrm{~A})$ & $1.613(2)$ \\
$\mathrm{Si}(8 \mathrm{~A})-\mathrm{O}(7 \mathrm{~A})$ & $1.615(2)$ & $\mathrm{Si}(8 \mathrm{~A})-\mathrm{O}(8 \mathrm{~A})$ & $1.6241(17)$ \\
& \multicolumn{2}{c}{$\mathrm{Bond}$ angles $\left({ }^{\circ}\right)$} & \\
$\mathrm{O}(1 \mathrm{~A})-\mathrm{Si}(1 \mathrm{~A})-\mathrm{O}(8 \mathrm{~A})$ & $107.52(10)$ & $\mathrm{O}(6 \mathrm{~A})-\mathrm{Si}(6 \mathrm{~A})-\mathrm{O}(9 \mathrm{~A})$ & $109.79(9)$ \\
$\mathrm{O}(1 \mathrm{~A})-\mathrm{Si}(1 \mathrm{~A})-\mathrm{O}(9 \mathrm{~A})$ & $110.38(9)$ & $\mathrm{O}(6 \mathrm{~A})-\mathrm{Si}(7 \mathrm{~A})-\mathrm{O}(7 \mathrm{~A})$ & $108.45(10)$ \\
$\mathrm{O}(8 \mathrm{~A})-\mathrm{Si}(1 \mathrm{~A})-\mathrm{O}(9 \mathrm{~A})$ & $109.70(10)$ & $\mathrm{O}(7 \mathrm{~A})-\mathrm{Si}(8 \mathrm{~A})-\mathrm{O}(8 \mathrm{~A})$ & $108.31(10)$ \\
$\mathrm{O}(1 \mathrm{~A})-\mathrm{Si}(2 \mathrm{~A})-\mathrm{O}(2 \mathrm{~A})$ & $106.79(9)$ & $\mathrm{Si}(1 \mathrm{~A})-\mathrm{O}(1 \mathrm{~A})-\mathrm{Si}(2 \mathrm{~A})$ & $157.15(14)$ \\
$\mathrm{O}(1 \mathrm{~A})-\mathrm{Si}(2 \mathrm{~A})-\mathrm{O}(10 \mathrm{~A})$ & $109.41(10)$ & $\mathrm{Si}(1 \mathrm{~A})-\mathrm{O}(8 \mathrm{~A})-\mathrm{Si}(8 \mathrm{~A})$ & $149.86(14)$ \\
$\mathrm{O}(2 \mathrm{~A})-\mathrm{Si}(2 \mathrm{~A})-\mathrm{O}(10 \mathrm{~A})$ & $109.15(9)$ & $\mathrm{Si}(1 \mathrm{~A})-\mathrm{O}(9 \mathrm{~A})-\mathrm{Si}(6 \mathrm{~A})$ & $147.26(11)$ \\
$\mathrm{O}(2 \mathrm{~A})-\mathrm{Si}(4 \mathrm{~A})-\mathrm{O}(3 \mathrm{~A})$ & $110.55(10)$ & $\mathrm{Si}(2 \mathrm{~A})-\mathrm{O}(2 \mathrm{~A})-\mathrm{Si}(3 \mathrm{~A})$ & $140.34(10)$ \\
$\mathrm{O}(3 \mathrm{~A})-\mathrm{Si}(4 \mathrm{~A})-\mathrm{O}(4 \mathrm{~A})$ & $108.50(9)$ & $\mathrm{Si}(2 \mathrm{~A})-\mathrm{O}(10 \mathrm{~A})-\mathrm{Si}(5 \mathrm{~A})$ & $140.11(11)$ \\
$\mathrm{O}(4 \mathrm{~A})-\mathrm{Si}(5 \mathrm{~A})-\mathrm{O}(5 \mathrm{~A})$ & $106.88(9)$ & $\mathrm{Si}(4 \mathrm{~A})-\mathrm{O}(3 \mathrm{~A})-\mathrm{Si}(3 \mathrm{~A})$ & $152.04(12)$ \\
$\mathrm{O}(4 \mathrm{~A})-\mathrm{Si}(5 \mathrm{~A})-\mathrm{O}(10 \mathrm{~A})$ & $109.09(10)$ & $\mathrm{Si}(5 \mathrm{~A})-\mathrm{O}(4 \mathrm{~A})-\mathrm{Si}(4 \mathrm{~A})$ & $142.70(12)$ \\
$\mathrm{O}(5 \mathrm{~A})-\mathrm{Si}(5 \mathrm{~A})-\mathrm{O}(10 \mathrm{~A})$ & $109.32(9)$ & $\mathrm{Si}(5 \mathrm{~A})-\mathrm{O}(5 \mathrm{~A})-\mathrm{Si}(6 \mathrm{~A})$ & $146.08(12)$ \\
$\mathrm{O}(5 \mathrm{~A})-\mathrm{Si}(6 \mathrm{~A})-\mathrm{O}(6 \mathrm{~A})$ & $108.12(9)$ & $\mathrm{Si}(6 \mathrm{~A})-\mathrm{O}(6 \mathrm{~A})-\mathrm{Si}(7 \mathrm{~A})$ & $150.43(13)$ \\
$\mathrm{O}(5 \mathrm{~A})-\mathrm{Si}(6 \mathrm{~A})-\mathrm{O}(9 \mathrm{~A})$ & $109.03(10)$ & $\mathrm{Si}(7 \mathrm{~A})-\mathrm{O}(7 \mathrm{~A})-\mathrm{Si}(8 \mathrm{~A})$ & $166.54(12)$ \\
\hline
\end{tabular}




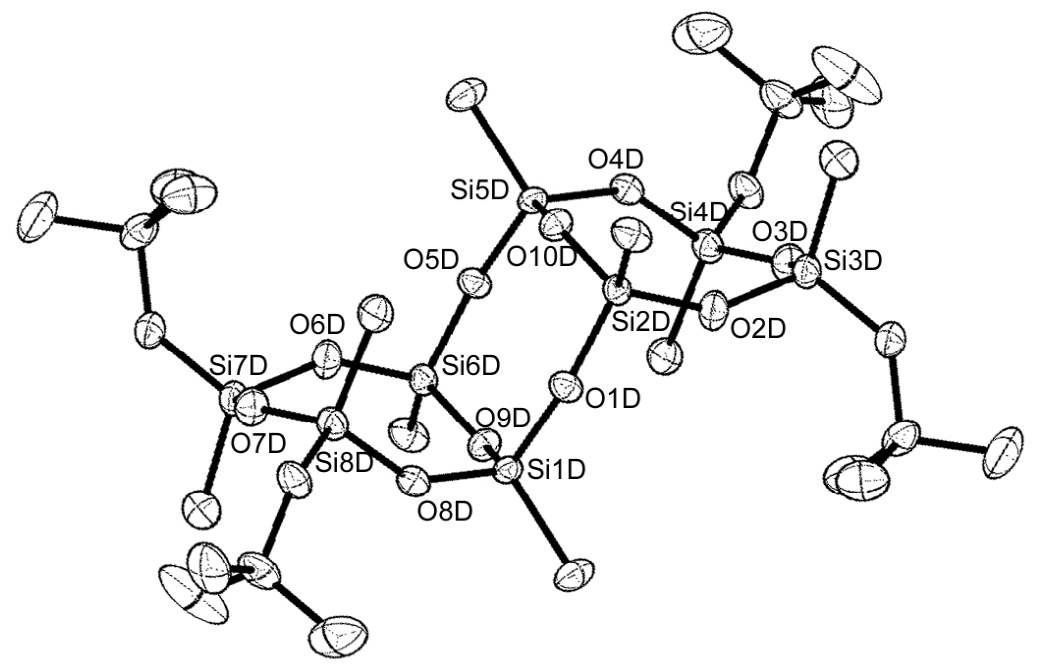

Figure S3. ORTEP drawing of 5d. Thermal ellipsoids are drawing at the $50 \%$ probability level. Hydrogen atoms are omitted for clarity.

Table S3. Selected bond lengths and angles of 5d

\begin{tabular}{|c|c|c|c|}
\hline \multicolumn{4}{|c|}{ Bond lengths $(\AA)$} \\
\hline $\mathrm{Si}(1 \mathrm{D})-\mathrm{O}(1 \mathrm{D})$ & $1.6212(19)$ & $\mathrm{Si}(5 \mathrm{D})-\mathrm{O}(4 \mathrm{D})$ & $1.621(2)$ \\
\hline $\mathrm{Si}(1 \mathrm{D})-\mathrm{O}(8 \mathrm{D})$ & $1.621(2)$ & $\mathrm{Si}(5 \mathrm{D})-\mathrm{O}(5 \mathrm{D})$ & $1.6212(19)$ \\
\hline $\mathrm{Si}(1 \mathrm{D})-\mathrm{O}(9 \mathrm{D})$ & $1.625(2)$ & $\mathrm{Si}(5 \mathrm{D})-\mathrm{O}(10 \mathrm{D})$ & $1.625(2)$ \\
\hline $\mathrm{Si}(2 \mathrm{D})-\mathrm{O}(1 \mathrm{D})$ & $1.6170(19)$ & $\mathrm{Si}(6 \mathrm{D})-\mathrm{O}(5 \mathrm{D})$ & $1.6170(19)$ \\
\hline $\mathrm{Si}(2 \mathrm{D})-\mathrm{O}(2 \mathrm{D})$ & $1.616(2)$ & $\mathrm{Si}(6 \mathrm{D})-\mathrm{O}(6 \mathrm{D})$ & $1.616(2)$ \\
\hline $\mathrm{Si}(2 \mathrm{D})-\mathrm{O}(10 \mathrm{D})$ & $1.625(2)$ & $\mathrm{Si}(6 \mathrm{D})-\mathrm{O}(9 \mathrm{D})$ & $1.625(2)$ \\
\hline $\mathrm{Si}(3 \mathrm{D})-\mathrm{O}(2 \mathrm{D})$ & $1.632(2)$ & $\mathrm{Si}(7 \mathrm{D})-\mathrm{O}(6 \mathrm{D})$ & $1.632(2)$ \\
\hline $\mathrm{Si}(3 \mathrm{D})-\mathrm{O}(3 \mathrm{D})$ & $1.625(2)$ & $\mathrm{Si}(7 \mathrm{D})-\mathrm{O}(7 \mathrm{D})$ & $1.625(2)$ \\
\hline $\mathrm{Si}(4 \mathrm{D})-\mathrm{O}(3 \mathrm{D})$ & $1.629(2)$ & $\mathrm{Si}(8 \mathrm{D})-\mathrm{O}(7 \mathrm{D})$ & $1.629(2)$ \\
\hline $\mathrm{Si}(4 \mathrm{D})-\mathrm{O}(4 \mathrm{D})$ & $1.634(2)$ & $\mathrm{Si}(8 \mathrm{D})-\mathrm{O}(8 \mathrm{D})$ & $1.634(2)$ \\
\hline \multicolumn{4}{|c|}{ Bond angles $\left({ }^{\circ}\right)$} \\
\hline O(1D)-Si(1D)-O(8D) & $108.56(10)$ & $\mathrm{O}(5 \mathrm{D})-\mathrm{Si}(6 \mathrm{D})-\mathrm{O}(9 \mathrm{D})$ & $109.46(10)$ \\
\hline O(1D)-Si(1D)-O(9D) & $109.34(10)$ & O(6D)-Si(7D)-O(7D) & $107.51(11)$ \\
\hline $\mathrm{O}(8 \mathrm{D})-\mathrm{Si}(1 \mathrm{D})-\mathrm{O}(9 \mathrm{D})$ & $109.33(10)$ & $\mathrm{O}(7 \mathrm{D})-\mathrm{Si}(8 \mathrm{D})-\mathrm{O}(8 \mathrm{D})$ & $107.55(11)$ \\
\hline $\mathrm{O}(1 \mathrm{D})-\mathrm{Si}(2 \mathrm{D})-\mathrm{O}(2 \mathrm{D})$ & $107.59(11)$ & $\mathrm{Si}(1 \mathrm{D})-\mathrm{O}(1 \mathrm{D})-\mathrm{Si}(2 \mathrm{D})$ & $147.75(13)$ \\
\hline $\mathrm{O}(1 \mathrm{D})-\mathrm{Si}(2 \mathrm{D})-\mathrm{O}(10 \mathrm{D})$ & $109.46(10)$ & $\mathrm{Si}(1 \mathrm{D})-\mathrm{O}(9 \mathrm{D})-\mathrm{Si}(6 \mathrm{D})$ & $144.24(13)$ \\
\hline $\mathrm{O}(2 \mathrm{D})-\mathrm{Si}(2 \mathrm{D})-\mathrm{O}(10 \mathrm{D})$ & 109.13(10) & $\mathrm{Si}(1 \mathrm{D})-\mathrm{O}(8 \mathrm{D})-\mathrm{Si}(8 \mathrm{D})$ & $143.27(13)$ \\
\hline $\mathrm{O}(2 \mathrm{D})-\mathrm{Si}(3 \mathrm{D})-\mathrm{O}(3 \mathrm{D})$ & 107.51(11) & $\mathrm{Si}(2 \mathrm{D})-\mathrm{O}(2 \mathrm{D})-\mathrm{Si}(3 \mathrm{D})$ & $148.74(15)$ \\
\hline $\mathrm{O}(3 \mathrm{D})-\mathrm{Si}(4 \mathrm{D})-\mathrm{O}(4 \mathrm{D})$ & $107.55(11)$ & $\mathrm{Si}(2 \mathrm{D})-\mathrm{O}(10 \mathrm{D})-\mathrm{Si}(5 \mathrm{D})$ & $144.24(13)$ \\
\hline O(4D)-Si(5D)-O(5D) & $108.56(10)$ & Si(3D)-O(3D)-Si(4D) & $141.98(14)$ \\
\hline $\mathrm{O}(4 \mathrm{D})-\mathrm{Si}(5 \mathrm{D})-\mathrm{O}(10 \mathrm{D})$ & $109.33(10)$ & Si(4D)-O(4D)-Si(5D) & $143.27(13)$ \\
\hline $\mathrm{O}(5 \mathrm{D})-\mathrm{Si}(5 \mathrm{D})-\mathrm{O}(10 \mathrm{D})$ & 109.34(10) & $\mathrm{Si}(5 \mathrm{D})-\mathrm{O}(5 \mathrm{D})-\mathrm{Si}(6 \mathrm{D})$ & $147.75(13)$ \\
\hline O(5D)-Si(6D)-O(6D) & $107.59(11)$ & Si(6D)-O(6D)-Si(7D) & $148.74(15)$ \\
\hline $\mathrm{O}(6 \mathrm{D})-\mathrm{Si}(6 \mathrm{D})-\mathrm{O}(9 \mathrm{D})$ & $109.13(10)$ & $\mathrm{Si}(7 \mathrm{D})-\mathrm{O}(7 \mathrm{D})-\mathrm{Si}(8 \mathrm{D})$ & $141.98(14)$ \\
\hline
\end{tabular}

